ÉGYPTE monde arabe

\section{Égypte/Monde arabe}

$2 \mid 1990$

Médiateur et métaphores 1

\title{
Deux formes de médiation partisane : islam traditionnel \& islam « moderniste » au Soudan
}

\section{Gamal Abd al-Gawad}

\section{(2) OpenEdition \\ 12 Journals}

Édition électronique

URL : https://journals.openedition.org/ema/215

DOI : 10.4000/ema. 215

ISSN : 2090-7273

\section{Éditeur}

CEDEJ - Centre d'études et de documentation économiques juridiques et sociales

\section{Édition imprimée}

Date de publication : 30 juin 1990

Pagination : 27-34

ISSN : 1110-5097

\section{Référence électronique}

Gamal Abd al-Gawad, « Deux formes de médiation partisane : islam traditionnel \& islam

« moderniste » au Soudan », Égypte/Monde arabe [En ligne], 2 | 1990, mis en ligne le 08 juillet 2008,

consulté le 07 juillet 2022. URL : http://journals.openedition.org/ema/215 ; DOI : https://doi.org/

10.4000/ema. 215

Ce document a été généré automatiquement le 7 juillet 2022.

Tous droits réservés 


\title{
Deux formes de médiation partisane : islam traditionnel \& islam « moderniste » au Soudan
}

\author{
Gamal Abd al-Gawad
}

\section{NOTE DE L'ÉDITEUR}

Traduit de l'arabe par Iman Farag

1 II semble important de préciser au départ que toute approche des phénomènes sociaux, culturels ou politiques au Soudan doit prendre comme point de départ la division de la société soudanaise en groupes ethniques différents qui prennent la forme de tribus, de lignages, de groupes linguistiques ou religieux.

2 Les études ethnographiques partent généralement de la tribu comme principale unité de formation sociale, du moins dans les régions peu exposées aux effets de la modernisation, puisqu'on retrace une configuration différente au niveau des villes. Cela ne veut pas dire pour autant que la tribu soit la seule forme d'unité sociale. D'autres formes existent certes, mais qui ne jouent pas le même rôle. C'est en effet à travers la tribu - unité de base de la solidarité sociale - que se joue la gestion de la vie quotidienne. Production et répartition des ressources, défense des individus, mais aussi un minimum de sentiment d'appartenance identitaire que la tribu donne à ses membres. Les autres niveaux d'identification ethnique (religieux, linguistiques) jouent plutôt un rôle de classification mais n'interviennent ni dans les interactions sociales ni dans la gestion du quotidien.

On sait par ailleurs que l'islam ne s'est pas diffusé, au Soudan, sur le mode conquérant qui a présidé à sa diffusion dans l'ensemble du Moyen-Orient et du monde arabe. Au Soudan, I'islam s'est ancré plus tardivement, par l'interaction naturelle de plusieurs facteurs : caravanes de commerce et déplacements de tribus de la Péninsule au Soudan, effort des prédicateurs musulmans... L'entrée de l'islam au Soudan correspond à une 
phase d'expansion importante du rôle des confréries à l'échelle des pays musulmans du Moyen-Orient. Certaines de ces confréries allaient avoir un rôle important dans l'islamisation du Soudan. C'est ce qui explique le fait qu'aujourd'hui encore, l'islam soudanais reste marqué par une dominante soufie et populaire et se distingue de l'islam sunnite, institutionnel et officiel de l'Égypte ou du Machrek.

4 Ce qu'il importe de souligner ici est que l'appartenance à une ou plusieurs confréries réunissait sous une même enseigne plusieurs tribus. Pour la première fois dans l'histoire du Soudan, les confréries soufis rendaient possible l'adhésion à un niveau d'appartenance supra-tribal et le regroupement de plusieurs tribus sur des bases autres que tribales. Cet impact n'était pourtant pas décisif dans la mesure où les tribus continuaient de gérer la vie quotidienne. Cependant, l'appartenance à la tribu était doublée d'une appartenance à un ensemble culturel plus large. Il en résultait de la sorte un type de "confédérations tribales » qui permettait d'assurer à la société soudanaise un minimum de cohésion.

L'émergence d'un islam communautariste

5 Cet état du fait est demeuré inchangé jusqu'à l'apparition du mouvement mahdiste, qui annonçait un tournant décisif. Muhammad Ahmad al-Mahdi se présentait comme alternative aux diverses confréries soufies, comme tentative d'unification, voire comme seule expression véridique de l'islam, le reste n'étant qu'hérésies. Cette crispation qui marque le mouvement mahdiste à ses débuts est due dans une large mesure à la nature même de la notion de mahdî qu'on retrouve également - soulignons le en passant - dans l'islam sunnite. Commença alors une période marquée par l'offensive du mouvement mahdiste, contre les confréries d'une part, contre le pouvoir turco-égyptien qui dominait le Soudan depuis le début du XIXe siècle, d'autre part.

6 Les confréries, elles, refusaient de se laisser assimiler dans I'lslam du mahdî et défendaient leur existence au sein de communautés indépendantes. C'est seulement à partir de ce moment qu'on peut parier d'un islam communautariste au Soudan. Celui-ci s'est développé dans le sillage de la réaction des confréries aux menaces du mouvement mahdiste. Jusqu'ici, l'appartenance soufie s'ajoutait à l'identification tribale mais la réponse au mahdisme s'exprima sous la forme d'une crispation communautariste et identitaire.

7 On sait ce qu'il en fut de la tentative du mouvement mahdiste : celui-ci échoua, tant à assimiler les confréries qu'à consolider son État. Dans la période qui suivit la chute de l'État mahdiste sous les attaques du pouvoir anglo-égyptien, entre 1898 et le début de ce siècle, le nouveau pouvoir créait une situation favorable aux confréries, dont les Anglais toléraient l'activité. Si elles gardaient la configuration acquise durant les périodes de conflit avec le mouvement mahdiste, ce dernier se devait d'introduire les modifications nécessaires à sa survie dans un contexte politique nouveau. Il s'agissait, pour ce mouvement, de se donner une image plus acceptable auprès des autorités britanniques. Cette reconversion n'a été possible qu'au prix d'un amoindrissement de la notion de mahdi elle-même, désormais mise en veilleuse. Le mouvement mahdiste renonçait à ses visées unitaristes et acceptait la pluralité communautaire de l'islam soudanais.

Un nouveau rapport au politique

8 Ce passage à une "real-politik» s'est notamment accompli avec 'Abd al-Rahman alMahdi, fils du fondateur, qui à son tour concevait un «néo-mahdisme » marqué par les exigences de l'adaptation à une situation nouvelle. Désormais, le mouvement des 
Ansars (partisans du mahdî) se repliait sur des bases sociales traditionnelles (à l'ouest du Soudan), renonçait à la violence et manifestait moins d'activité dans l'expansion de ses idées. En somme, le mahdisme perdait de ses caractéristiques comme mouvement de Jihâd.

En définitive, l'émergence d'un islam communautariste au Soudan est intimement lié à :

- la lutte des confréries contre le mouvement mahdiste,

- l'adaptation de ce mouvement face à de nouvelles exigences,

- un nouveau rapport au politique qui s'est instauré parmi les groupes communautaires, dans le contexte des aménagements que le mouvement des Ansars introduisait dans son idéologie et ses pratiques.

10 Ces groupes constituent la base sociale des principaux partis politiques au Soudan. La présence des confréries ne signifiait pas en elle-même une structuration communautariste. Ce n'est qu'à partir du moment ou elles ont manifesté un intérêt accru pour la politique - marquées en cela par l'expérience des Ansars - et qu'elles se sont redéfinies sur de nouvelles bases plus complexes qu'on a pu parler d'un islam communautariste.

11 Du côté des Ansars, on retrouvait toujours le leadership de type traditionnel représenté par 'Abd al-Rahman al-Mahdi. Mais ils acceptaient désormais de n'être qu'une communauté musulmane parmi d'autres, et non la Communauté des musulmans. A l'instar du mahdisme, les bases du néo-mahdisme se concentraient toujours à l'ouest et regroupaient trois principaux groupes linguistiques et lignagers différents ; les Arabes, les Fours et les Foulains. En ce sens, le mouvement avait réussi à intégrer l'ensemble des groupes ethniques différents sur la base d'une appartenance au mouvement mahdiste. En revanche la Khatimiyya - la confrérie la plus importante après le mouvement mahdiste, une des plus anciennes aussi, essentiellement établie au nord, à l'est et, dans une certaine mesure, au centre du Soudan - avait réussi à intégrer les Arabes du nord et du centre aux Bejas de l'Est soudanais, qui constituent un groupe ethnique et culturel distinct non arabophone. En ce sens, l'émergence d'un islam communautariste proposait une forme d'identification supérieure, au-delà des appartenances ethniques tribales et lignagères. Toutefois, ce découpage entre ouest, nord et centre avait un impact considérable sur les orientations des deux mouvements, notamment sur le plan culturel.

Etablie au nord et au centre, la Khatimiyya était plus exposée aux effets d'une culture islamique arabe ou méditerranéenne via l'Égypte. Chez les Ansars, établis à l'ouest, les interactions étaient plus importantes en direction de l'Afrique de l'ouest, allant jusqu'au Tchad, au Nigeria et au Sénégal.

Vers des partis modernes

13 Ces effets culturels différents marquent de manière très forte le mouvement politique soudanais. La constitution des partis politiques soudanais dans les années 40 est fortement marquée par le découpage entre le parti al-Umma, soutenu par le mouvement des Ansars, et les partis unitaristes qui formeront plus tard le Parti national unitaire (Ittihâdî) et dont la base sociale est principalement la confrérie Khatimiyya. Dans ce contexte nouveau de politique nationale, ces groupes tentent d'acquérir la configuration de partis modernes. L'heure n'est pas aux conflits intertribaux mais à la compétition pour un pouvoir politique, compétition qui, par définition, est ouverte à tous, puisque le pouvoir est censé couvrir et représenter 
l'ensemble du pays. Durant toute la période qui va jusqu'à l'acquisition de l'indépendance, les deux partis al-Umma et al-Ittihâdî tentent ainsi de se redéfinir en tant que partis nationaux.

Riche de son histoire anti-coloniale, des luttes menées entre l'occupant turc, égyptien ou britannique et faisant valoir son expérience de constitution du premier État soudanais, le parti al-Umma se présente comme symbole d'un nationalisme soudanais. Ne pouvant prétendre à un passé aussi riche, le parti al-Ittihâdî compte sur le soutien des couches moyennes modernes appartenant à différents groupes ethniques et tribaux et se revendique lui aussi d'un nationalisme soudanais. Toutefois, aucun des deux partis n'a été en mesure de disputer à son concurrent ses assises sociales, et cet état de fait renforçait encore le caractère communautariste des partis politiques soudanais. Notons à cet égard que les deux partis ont été incapables de réaliser la moindre percée dans le Sud, et ont ainsi limité leur influence aux Soudanais musulmans. Cela explique dans une large mesure, les échecs successifs des tentatives de constitution d'un pouvoir démocratique au Soudan.

Cependant, des mutations profondes allaient marquer la configuration socio-politique soudanaise exposée aux effets de la modernisation. Celle-ci est repérable à plusieurs niveaux: développement de l'enseignement, constitution des appareils d'État modernes, moyens de communication, modernisation économique - notamment dans l'agriculture avec le projet de la Guezira -, émergence d'un secteur industriel et bancaire. Par la conjugaison de ces facteurs, des couches de plus en plus importantes se retrouvaient libérées des appartenances tribales et communautaires. Concentrées essentiellement dans les villes et au centre du Soudan, les nouvelles couches - «les diplômés » dans la terminologie soudanaise - ont tenté de mettre en place des cadres autonomes d'expression politique, indépendamment des partis communautaristes. Parmi ces tentatives, il faut noter celle du courant communiste implanté au centre dans les régions les plus modernisées, mais aussi celle de l'islamisme politique qui s'annonce dès les années 40 , se manifeste de façon plus claire dans les années 50 et 60 sous le vocable «Front de la charte islamique » et constitue dans les années 80 «le Front islamique national ». Il s'agit là d'un parti politique à part entière, en compétition pour le pouvoir. Si cette compétition donne lieu à des alliances avec les partis communautaristes et traditionnels, il n'en demeure pas moins que le mouvement islamiste se situe clairement dans une visée moderniste. Le communautarisme est ainsi dénoncé comme signe et cause du «retard» soudanais. Sur le plan idéologique, ce mouvement ne diffère en rien, à ses débuts du moins, des divers mouvements fondamentalistes, notamment en ce qui concerne l'opposition aux confréries, réfutées tant sur le plan religieux - « hérésies » - que politique - « division de la Umma ».

C'est aux efforts de Hasan al-Turabi que l'on doit les principaux éléments de modernisation de l'islam politique soudanais. Les plus significatifs portent sur le renouvellement du fiqh, que Turabi a poussé beaucoup plus loin que l'ensemble des mouvements islamistes. Si ces derniers s'attachent - quand ils le font - au renouvellement des furû' («branches»), Turabi va jusqu'à discuter les usûl (" sources »). Pour lui l'Ijtihâd (exégèse, interprétation) est ouverte à tous sans critères de distinction sociale, voire scientifique. Un ensemble souple de paramètres devrait permettre d'évaluer la justesse de l'Ittihâd et non de l'interdire, et c'est à l'ensemble des musulmans que revient en dernière instance le droit d'évaluation. On peut noter ici une avancée notoire par rapport aux Frères Musulmans égyptiens. Il est clair, 
cependant, que le but de l'opération est de casser le monopole des leaders communautaristes dans ce domaine.

17 A un autre niveau, l'islamisme soudanais se distingue par sa position à l'égard des femmes, qui a d'ailleurs considérablement influencé celle des islamistes tunisiens. Les islamistes soudanais encouragent l'enseignement supérieur pour les femmes et sont favorables à ce qu'elles travaillent, y compris dans les fonctions supérieures et la magistrature. Aux élections de l'Assemblée constitutionnelle en 1986, trois candidates ont été élues sur les listes du Front islamique.

Au niveau des pratiques, on peut constater une distanciation par rapport à tout ce qui représente l'islam traditionnel: des vêtements modernes, une structure de parti des plus achevées, des filières économiques et financières d'une remarquable efficacité et construites sur les modèles occidentaux. Faisant appel à une idéologie vieille de quatorze siècles, le mouvement islamiste soudanais n'en est pas moins farouchement opposé à toutes les structures qu'il considère comme "anti-modernes ». Il recrute parmi les catégories sociales modernes, obtient, aux élections de 1986, 23 des 28 sièges destinés aux "diplômés » et dont les circonscriptions sont pour la plupart urbaines. Quant aux 31 circonscriptions de Khartoum, le Front islamique réussit à en obtenir 13. Déjà, en 1965 et 1968, les islamistes soudanais avaient réussi à emporter trois sièges dans les circonscriptions de la capitale et les régions modernisées de la Guezira.

Le jeu des alliances politiques

En dépit de ses succès, le Front islamique ne pouvait rester limité aux seules couches modernes. Pour se transformer en mouvement de masse et s'ouvrir à d'autres catégories sociales, il lui fallait élaborer une autre approche des réalités soudanaise et plus particulièrement de l'islam traditionnel. C'est pendant la phase de leur alliance avec l'ancien président Nemeyri que les islamistes ont mis en place un nouveau langage. Désormais, la coopération avec les tribus et les confréries était admise dans la mesure ou ces forces - aussi traditionnelles soient-elles - seraient prêtes à soutenir le mouvement islamiste.

Par ailleurs, l'islam populaire et confrérique n'était plus dénoncé comme une hérésie. Le Front islamique avait compris que pour se transformer en mouvement de masse, il ne fallait plus raisonner en termes de recrutement des individus dans une société tribale, puisque dans une telle société - à l'exception des catégories modernes l'individu n'existe pas par définition. Il fallait donc s'adresser à des groupes. C'est la prise en compte de cette réalité qui a permis au mouvement islamiste de sortir de l'enclave villes/catégories urbaines modernes et de s'étendre au monde rural et aux zones peu touchées par la modernisation.

21 Par ailleurs, le mouvement islamiste est parvenu à tirer parti des contradictions internes de ses concurrents. Il en est ainsi, par exemple, des tensions entre les groupes fours et arabes qui soutenaient le parti al-Umma. En s'affirmant comme parti politique capable de prendre en charge des revendications, le Front islamique a pu obtenir le soutien de la majorité des Fours et emporta pour la première fois des sièges dans la région du Darfour. A un troisième niveau, enfin, le Front islamique réussit, par son réseau de banques et de sociétés islamiques implanté sous Nemeyri, à acheter le soutien de plusieurs tribus moyennant aide financière.

C'est grâce à ces divers dispositifs que le Front islamique a pu obtenir en 1986 - en plus des sièges destinés aux diplômés - 28 circonscriptions géographiques sur l'ensemble des régions et tribus soudanaises, résultat que seuls des partis communautaristes 
avaient jusqu'ici réussi à obtenir. Ainsi le Front islamique réussissait-il à s'octroyer non seulement le soutien des nouvelles couches moyennes mais aussi celui d'une part importante du secteur traditionnel.

Cette dernière percée s'est effectuée aux dépens du parti al-Ittihâdi et de ses zones d'implantation traditionnelles. A Khartoum, le Front a remporté 11 des circonscriptions d'al-Ittihâdi dans le Nord, 3 d'al-Ittihâdi et une d'al-Umma, et 3 également dans la région du Centre. Le fait que cette percée se soit produite principalement contre le parti al-lttihâdi est dû dans une large mesure aux faiblesses internes de ce parti et de la confrérie Khatimiyya, alors que le parti al-Umma et le mouvement des Ansars se caractérisent par une organisation plus rigoureuse.

Il faut également prendre en compte l'ouverture du mouvement islamiste soudanais aux influences égyptiennes, notamment celles des Frères Musulmans, même s'il s'en est démarqué par la suite. Du fait de cet impact culturel, on peut dire que le Front islamique parlait une langue qui n'était pas totalement étrangère au public de la Khatimiyya dans le centre et le nord. Et c'est peut-être en partie grâce à cela que le Front islamique a pu disputer au parti al-Ittihâdi son public, de manière plus efficace qu'il n'a pu le faire en direction des Ansars.

Ce qu'il importe de souligner ici est que le mouvement a cédé devant le communautarisme en adoptant la logique de la division, que les discours idéologiques ne permettaient pas de transcender. En revanche, le Front islamique parvenait à réunir à nouveau et dans le cadre de "l'islam» des tribus et des confréries différentes. Ce n'est pas par hasard, d'ailleurs, que le mouvement se donne le nom de "Front", signifiant par là son ouverture à plusieurs factions: les Frères Musulmans, les confréries soufies et autres groupes font désormais partie de ce " Front », qui n'est plus le "parti des Frères" ni la "charte islamique ». Ce qui se joue est en somme une " répétition ». Les mahdistes avaient été contraints de se redéfinir comme communauté parmi d'autres (et non comme la Communauté); le mouvement islamiste doit, à sa manière, prendre en compte les réalités d'une société tribale.

Cette prise en compte, et la capacité du Front à rogner sur la base sociale des partis traditionnels, poussant ainsi cette base à se redéfinir elle-même selon de nouveaux critères d'appartenance, n'a pas été sans effets au niveau des partis communautaristes eux-mêmes. Cela s'est manifesté de façon plus claire en ce qui concerne le parti alUmma, notamment avec la fin des leaders traditionnels et le leadership de Sadiq alMahdi. Maîtrisant la culture occidentale, ayant suivi un cursus d'enseignement moderne, Sadiq al-Mahdi a tenté de donner à son mouvement un visage plus acceptable. Ses écrits des années 70 tentent de situer le mouvement mahdiste dans le contexte de la montée des mouvements islamistes. Il s'agirait donc d'un mouvement renouvelé, moderne et non d'un mouvement communautariste du siècle dernier. Par ce biais, Sadiq al-Mahdi a réussi en effet à s'attirer un soutien important de la part des catégories urbaines et modernes. Aux élections de 1986, il a réussi à obtenir pour la première fois 6 circonscriptions de la capitale, contre une seule aux élections précédentes.

27 En revanche, le cas du parti al-Ittihâdi et de la Khatimiyya est le type même de l'échec total de l'adaptation aux exigences d'une nouvelle société. Société tribale certes, mais en voie de modernisation et qui, pour ces raisons mêmes aurait besoin, davantage encore, de se redéfinir. Le parti al-Ittihâdi a perdu ses dirigeants historiques dans une période très brève. Ils appartiennent pour la plupart au profil des leaders traditionnels. 
Bien loin de ressembler à Sadiq al-Mahdi, Muhammad al-Mirghani, leader actuel d'alIttihâdi, ressemble à son père. Il en est la simple reproduction.

En ce sens, on peut examiner le rapport entre islam traditionnel et islam moderne en partant d'un point précis (qui est aussi un processus). A un moment donné, la communauté a besoin d'un leadership capable de formuler un nouveau cadre de référence et d'appartenance socio-politique. Cela est d'autant plus vrai quand il s'agit d'une société marquée par un caractère traditionnel (la tribu comme unité d'appartenance sociale) et soumise néanmoins aux effets de la modernisation (qui produisent notamment des "éduqués", les seuls en mesure de mener une lutte politique):

II semble que le succès d'un mouvement politique, qu'il soit communautaire ou moderne, est lié à sa capacité à proposer un cadre qui permet de faire la synthèse du moderne et du traditionnel. C'est ce qu'a produit le mouvement islamiste au Soudan. C'est ce qu'a produit également un parti traditionnel comme al-Umma.

INDEX

Mots-clés : islam, Soudan, religion, islam et politique

\section{AUTEUR}

GAMAL ABD AL-GAWAD

Centre d'études stratégiques d'Al-Ahram 